\title{
Otimização do processo de cristalização em cristalizador contínuo para fondant
} Optimization of the crystallization process in a continuous beater for Fondants

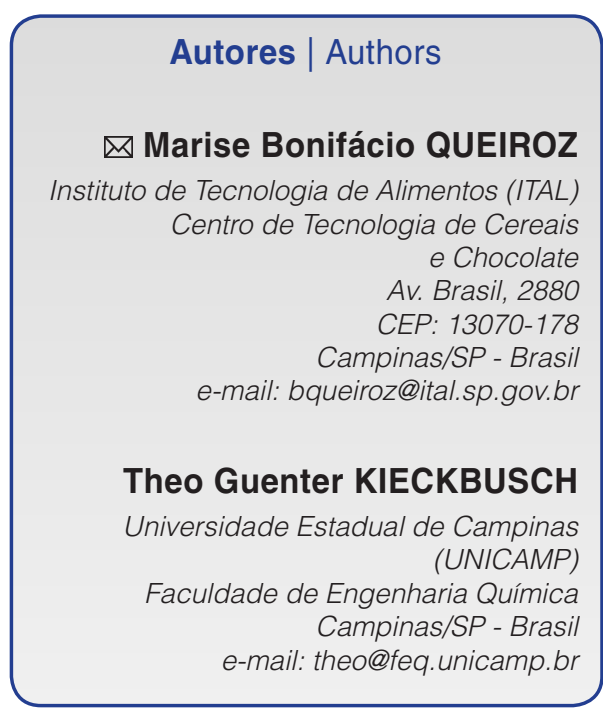

$\triangle$ Autor Correspondente / Corresponding Author

Recebido / Received: 02/03/2011 Aprovado / Approved: 23/04/2012 Publicado / Published: dez./2012

\section{Resumo}

Os principais fatores que afetam a textura em um fondant são: formulação, relacionada com a existência de outros ingredientes além da sacarose; temperatura de cristalização; geometria do cristalizador, e velocidade de cristalização. Neste estudo, procurou-se avaliar a influência de parâmetros de processo sobre as características texturais e de atividade de água de um fondant produzido com xarope de glicose obtido pela hidrólise da fécula de mandioca, utilizando-se um protótipo de cristalização contínuo com capacidade para 1 a $2 \mathrm{~kg} \cdot \mathrm{h}^{-1}$. Este equipamento foi projetado e construído baseado em modelo de equipamento contínuo para a produção industrial de fondant, sendo configurado com um sistema de rosca sem fim, encamisada para circulação de água fria. Selecionaram-se, para seu dimensionamento, parâmetros, como tamanho da rosca (comprimento, diâmetro e passo) e espaçamento (gap) entre a rosca e o tubo externo; para permitir variações na velocidade de rotação da rosca, optou-se pela instalação de um inversor de frequência acoplado ao motor. Amostras de fondant foram produzidas a partir de misturas de sacarose e xarope de fécula de mandioca 42DE, estudando-se a influência das variáveis temperatura de cristalização e velocidade de rotação sobre sua textura, e a atividade de água, utilizando-se delineamento estatístico Composto Central Rotacional com base na Metodologia de Superfície de Resposta (MSR). Com o uso do protótipo dotado de rosca de $250 \mathrm{~mm}$ de comprimento e 27,2 $\mathrm{mm}$ de diâmetro, e considerando-se as repostas de atividade de água e textura, concluiu-se que para obter as melhores características no fondant, com textura adequada e maior estabilidade microbiológica, deve-se trabalhar com teores de xarope de glicose de, no mínimo, 20\% (mínima atividade de água), velocidade de rotação entre 105 e 110 rpm, e temperatura inicial de cristalização ao redor de $60{ }^{\circ} \mathrm{C}$.

Palavras-chave: Fondant; Cristalização; Xarope de fécula de mandioca; Cristalizador contínuo; Textura.

\section{Summary}

The main factors affecting the texture of a fondant are its formulation, related to the presence of other ingredients besides sucrose, the crystallization temperature, beater design and beater speed. This investigation evaluated the influence of process parameters on the textural characteristics and water activity of fondants produced with glucose syrup obtained by hydrolyzing manioc starch. A small-scale prototype of an industrial beater with a capacity for 1 to $2 \mathrm{~kg} \cdot \mathrm{h}^{-1}$ was designed and tested. It consisted of a double jacketed cylinder containing a screw conveyor. The screw size (diameter, length and path), gap between the screw and the external tube and the range of screw rotation (a frequency inverter was coupled to the motor to allow for speed changes) were used as design criteria. Samples of fondant formulated with sucrose and 42DE manioc starch syrup were produced, and the influence of the crystallization temperature and rotation speed on the texture and water activity evaluated by a central composite design based on the fit to a second-order polynomial model, using response surface methodology. The results indicated that, considering the responses for water activity and texture, the use of the fondant beater equipped with a $250 \mathrm{~mm}$ length screw with a $27.2 \mathrm{~mm}$ diameter, allowed one to obtain an appropriate texture and microbiological stability using manioc starch syrup levels of at least $20 \%$ (minimum water activity), rotation speed between 105 and $110 \mathrm{rpm}$ and an initial beating temperature of $60{ }^{\circ} \mathrm{C}$.

Key words: Fondant; Crystallization; Cassava syrup; Continuous beater; Texture. 


\section{Introdução}

O fondant é o mais simples dos confeitos cristalizados de açúcar, consistindo de uma fase líquida, solução saturada de sacarose e outros carboidratos, e uma fase sólida cristalina, dispersão de pequenos cristais de sacarose (JEFFERY, 2001). Por ser um produto concentrado até a saturação, possui forte tendência à cristalização, sendo que esta deve ser controlada quanto ao tamanho e ao número de cristais gerados. A formação dos microcristais é induzida por uma intensa agitação (cristalização) de um xarope de açúcar supersaturado (JEFFERY,1993).

Muitos fatores influenciam a cristalização em um fondant, os quais podem ser divididos em fatores de formulação e fatores de processo. A formulação, incluindo a quantidade de água no xarope de glicose e a relação sacarose/xarope, afeta tanto as curvas no diagrama de estado como a cinética de nucleação/cristalização a uma dada condição de processo. O xarope é adicionado ao fondant, entre outras funções, para controlar a textura, fornecendo uma característica de plasticidade e aceitabilidade sensorial própria ao produto. Esse efeito deve-se à diminuição no volume da fase cristalina e ao aumento da concentração de sólidos em solução com o aumento do conteúdo relativo do xarope de glicose na formulação, assim como à diminuição do tamanho médio dos cristais formados durante a cristalização (HARTEL, 2001; HARTEL e SHASTRY, 1991).

As taxas de evaporação de água e de resfriamento, a temperatura de cristalização e a intensidade da agitação mecânica durante a cristalização são fatores de processo que podem ser ajustados para obter os atributos desejáveis de um fondant.

A literatura técnica ou científica não registra nenhuma informação sobre critérios de dimensionamento de um cristalizador em escala piloto. Uma referência com detalhes de projeto de um cristalizador em escala industrial foi encontrada em Vilchez et al. (2006), o qual detalha o esquema de um cristalizador patenteado pela empresa Klockner Hansel Proc Gmbh da Alemanha, configurado com um sistema de rosca que transporta o xarope de açúcares e induz sua cristalização, sendo dotado de um tubo (estator) com refrigeração externa.

Com o objetivo de estudar o processo de cristalização de uma massa de fondant em condições dinâmicas, próximas ao processo utilizado industrialmente, foi projetado, construído e posto em operação um cristalizador piloto, baseado em modelos de equipamentos contínuos para a produção industrial de fondant. Esses equipamentos, geralmente, são configurados com um sistema de rosca sem fim encamisada com circulação de água fria e o equipamento piloto projetado, neste estudo, seguiu o mesmo princípio de funcionamento, tendo a característica de desmontagem completa com facilidade de limpeza.

\section{Material e métodos}

\subsection{Projeto e construção do cristalizador}

Baseado em informações de equipamentos disponíveis industrialmente, como os de fabricação alemã da marca Chocotech, modelo Princess ${ }^{\circledR}$, com capacidade para $10 \mathrm{~kg} \cdot \mathrm{h}^{-1} \mathrm{e} \mathrm{o}$ industrial para uma capacidade de 400-2.000 kg. $\mathrm{h}^{-1}$, modelo Micron ${ }^{\circledR}$, do mesmo fabricante, propôs-se o projeto de um protótipo de cristalização com capacidade para 1 a $2 \mathrm{~kg} \cdot \mathrm{h}^{-1}$, selecionando-se em seu dimensionamento parâmetros, como tamanho da rosca (comprimento, diâmetro e passo) e gap entre a rosca e o tubo interno, entre outros. Os parâmetros do dimensionamento proposto para o cristalizador encontram-se na Tabela 1.

A Figura 1 mostra o esquema do projeto para o cristalizador, destacando detalhes de sua geometria e dimensões.

O equipamento foi construído pela empresa Valcamp - Válvulas e Acessórios para Laboratórios Ltda - ME, de Campinas-SP.

As vedações da rosca e da respectiva camisa de resfriamento foram feitas utilizando-se anéis de borrachas. Todos os outros componentes do equipamento foram construídos em aço-inoxidável, SS-316, com exceção

Tabela 1. Dimensionamento do cristalizador piloto.

\begin{tabular}{lll}
\multicolumn{1}{c}{ Rosca } & \multicolumn{1}{c}{ Tubo interno } & \multicolumn{1}{c}{ Tubo externo } \\
Comprimento $=250 \mathrm{~mm}$ & Diâmetro ext. $=33,4 \mathrm{~mm}$ & Diâmetro ext. $=60,4 \mathrm{~mm}$ \\
Diâmetro ext. $=27,2 \mathrm{~mm}$ & Diâmetro int. $=28 \mathrm{~mm}$ & Diâmetro int. $=54,8 \mathrm{~mm}$ \\
Diâmetro int. $=22,8 \mathrm{~mm}$ & \\
Altura $=2,2 \mathrm{~mm}$ & \\
Largura dente $=3,2 \mathrm{~mm}$ & \\
Espaço entre dentes $=2,7 \mathrm{~mm}$ & \\
Passo $=6,0 \mathrm{~mm}$ & \\
Gap $=0,8 \mathrm{~mm}$ &
\end{tabular}


da válvula de alimentação do xarope, que foi construída em alumínio.

Após a construção, o equipamento foi montado e fixado em base de madeira, procedendo-se à sua instalação elétrica. O motor foi instalado no eixo da rosca por meio de polias com canal em "V" e correia de borracha trapezoidal, e um inversor de frequência foi acoplado a fim de possibilitar a variação de velocidade de rotação da rosca durante o estudo de cristalização.

Ao equipamento, foi acoplado um banho termostatizado (Marca Marconi; Modelo: MA-184) para circulação de água fria pela camisa da rosca, a qual foi realizada em contracorrente ao fluxo da massa no interior da rosca.

Para manter constante a temperatura de cristalização, variável estudada na otimização da cristalização, foi instalada uma resistência elétrica (Tipo TR2 9325, 16A/250V, categoria IV) no fundo do tanque de alimentação do xarope, conforme observado na Figura 4c.

\subsection{Ensaios preliminares}

Com o equipamento montado, foram realizados ensaios de familiarização com os procedimentos, procurando definir o possível campo de variação de alguns parâmetros de processo e de produto (formulação), e avaliar o desempenho quanto aos seguintes aspectos: velocidade de rotação da rosca, temperatura da água de circulação da camisa, temperatura de cristalização (temperatura da alimentação do xarope) e temperatura de cozimento do xarope, além da concentração de xarope de fécula de mandioca na formulação.

Velocidade de rotação da rosca: a velocidade pré-selecionada no projeto do equipamento foi de 84 rpm. Os ensaios permitiram trabalhar com rotações variando na faixa de 65 a 135 rpm;

Temperatura do banho (água de circulação na camisa da rosca): foram avaliadas temperaturas de 10 , 15 e $20^{\circ} \mathrm{C}$;

Temperatura de cozimento do xarope: fixou-se em $114^{\circ} \mathrm{C} \pm 0,5^{\circ} \mathrm{C}$ a temperatura para o cozimento do xarope, de acordo com resultados obtidos em estudo anterior, com um sistema de cristalização descontínuo (QUEIROZ et al., 2006);

Concentração de xarope de glicose na formulação: também tendo como referência resultados obtidos em estudo com cristalizador descontínuo (QUEIROZ et al., 2006), foram realizados ensaios com concentrações de 0, 5, 10, 20 e 25\%, utilizando-se na formulação xarope de glicose obtido pela hidrólise da fécula de mandioca 42DE, fornecido pela empresa Indemil/Yoki, como alternativa ao xarope de milho comumente utilizado nestes processos. A utilização do xarope obtido pela hidrólise da fécula de mandioca em substituição ao xarope de milho se mostrou interessante por ser um xarope com as mesmas características do xarope de milho e ser produzido com matéria-prima e tecnologia nacionais;

Temperatura de cristalização: variações na temperatura de cristalização, na faixa de 50 a $100{ }^{\circ} \mathrm{C}$ foram avaliadas.

\subsection{Otimização do processo}

Os resultados obtidos nos ensaios preliminares quanto ao comportamento do equipamento e às características do produto serviram de base para se proporem limites ao delineamento experimental.

Fixando-se a temperatura de circulação da água na camisa e o ponto de cozimento do xarope, as variáveis estudadas para a otimização do processo no cristalizador contínuo foram: porcentagem de xarope de fécula de mandioca na formulação, velocidade de rotação da rosca e temperatura de cristalização nos níveis de variação indicados na Tabela 2.

Utilizou-se o delineamento experimental com o planejamento fatorial completo $2^{3}$ com pontos axiais e centrais, e o experimento gerado consistiu de 17 ensaios, sendo oito combinações entre as três variáveis em dois níveis $\left(2^{3}\right)$, seis ensaios dos pontos axiais e três repetições do ponto central. Para a análise dos resultados, utilizou-se metodologia de superfície de resposta, segundo proposto por Box et al. (1978) e Barros Neto et al. (2001). Para o cálculo dos pontos axiais, utilizou-se a equação $\alpha=\left(2^{n}\right)^{1 / 4}$,

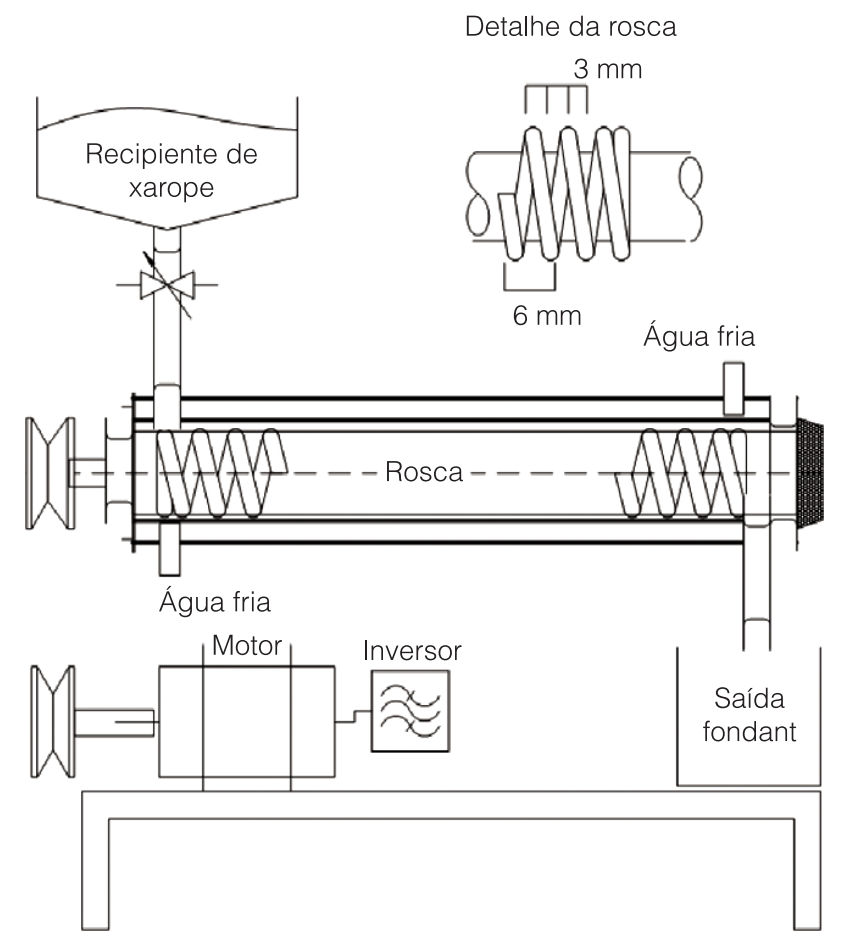

Figura 1. Desenho esquemático do cristalizador de fondant projetado com capacidade para 1 a $2 \mathrm{~kg} \cdot \mathrm{h}^{-1}$ (sem escala). 
na qual $n=$ número de variáveis, tendo-se $+\alpha=1,68$ e $-\alpha=-1,68$, para as variáveis codificadas.

Como variáveis de resposta ao delineamento, avaliaram-se a atividade de água e a textura instrumental da massa cristalizada, tendo-se como referência as seguintes metodologias:

Atividade de água (Aw): medida diretamente em analisador da Aw, de marca Decagon, modelo Aqua Lab $\mathrm{Cx}-2$ a $25^{\circ} \mathrm{C}$, e realizada em triplicata.

Textura objetiva: Utilizou-se uma adaptação da metodologia proposta pela Stable Micro Systems, específica para fondant, que consiste em determinação da firmeza/maciez da massa cristalina em função da força necessária para a compressão da amostra. Cada amostra de fondant foi distribuída em dez recipientes plásticos com tampa $(21 \mathrm{~mL}$, com 3,4 cm diâmetro e $2,2 \mathrm{~cm}$ altura) e armazenada em estufa incubadora à temperatura constante de $25^{\circ} \mathrm{C}$ por sete dias. Os valores de firmeza são médias de dez repetições para cada amostra. As condições para esta análise foram: módulo Força em Compressão, velocidade pré-teste 1,0 mm.s ${ }^{-1}$, velocidade de teste $2,0 \mathrm{~mm} \cdot \mathrm{s}^{-1}$, velocidade pós-teste 2,0 mm.s $\mathrm{s}^{-1}$, distância $15 \mathrm{~mm}$, trigger force - button/3 g, célula de carga $50 \mathrm{~kg}$ e probe cilíndrico $(4 \mathrm{~mm})$ de aço inoxidável.

\subsection{Procedimento experimental}

A produção das amostras de fondant foi feita seguindo o diagrama de blocos da Figura 2 e as etapas são descritas a seguir.

Mistura e dissolução: as quantidades pré-estabelecidas de água, sacarose e xarope de fécula de mandioca, de acordo com o delineamento experimental, foram pesadas e colocadas em um copo de aço-inox com capacidade para $2 \mathrm{~L}$ e diâmetro de $16 \mathrm{~cm}$. A solução foi aquecida em sistema à pressão atmosférica (fogo direto) até a completa dissolução dos açúcares ( $110^{\circ} \mathrm{C} / 80 \%$ sólidos), realizada sem agitação, por convecção natural e controlada com auxílio de um termômetro digital (Marca: Gulton, País: Brasil; Modelo: Gulterm 180; Precisão 0,1 ${ }^{\circ} \mathrm{C}$ );

Cozimento à pressão atmosférica: a solução foi concentrada em sistema de cozimento aberto (pressão atmosférica) em fogo direto, por convecção natural, até atingir a temperatura pré-estabelecida em $114{ }^{\circ} \mathrm{C}$, medida pelo mesmo termômetro digital; após cozida, foi transferida para o reservatório do protótipo de cristalização;

Resfriamento à pressão atmosférica: nesta fase, o xarope foi transferido para o reservatório do equipamento e deixado no ambiente até atingir temperatura definida para início da cristalização, sob condição de pressão atmosférica;

Cristalização/batimento: a cristalização foi realizada no protótipo de cristalização contínuo utilizando-se
Tabela 2. Níveis para as variáveis independentes utilizadas no estudo.

\begin{tabular}{lccccc}
\multicolumn{1}{c}{ Variáveis } & $-\alpha$ & $\mathbf{- 1}$ & $\mathbf{0}$ & $\mathbf{+ 1}$ & $\mathbf{+} \alpha$ \\
$\begin{array}{l}\text { Xarope de fécula de } \\
\text { mandioca (\%) }\end{array}$ & 0,92 & 5 & 11 & 17 & 21,08 \\
$\begin{array}{l}\text { Velocidade de rotação } \\
\text { da rosca (rpm) }\end{array}$ & 83,2 & 90 & 100 & 110 & 116,80 \\
$\begin{array}{l}\text { Temperatura de } \\
\text { cristalização }\left({ }^{\circ} \mathrm{C}\right)\end{array}$ & 53,2 & 60 & 70 & 80 & 86,80 \\
\hline
\end{tabular}

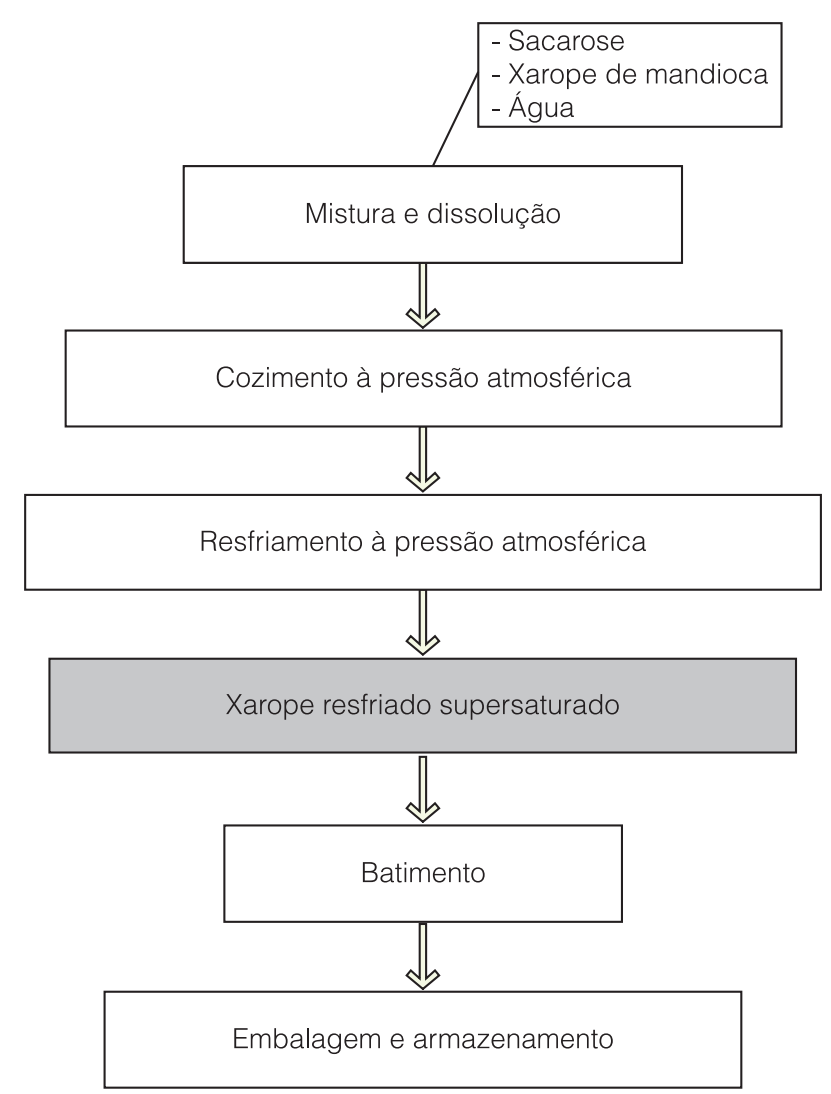

Figura 2. Diagrama de blocos para o processamento do fondant.

velocidade pré-determinada pelo delineamento experimental.

Embalagem e armazenamento: os fondants produzidos foram armazenados em vidros com tampa metálica e acondicionados em estufa BOD à temperatura constante de $25^{\circ} \mathrm{C}$ para posterior análise.

\section{Resultados e discussão}

\subsection{Construção do cristalizador}

As Figuras 3 e 4 mostram fotografias do equipamento montado e desmontado, enfocando detalhes de sua construção. 


\subsection{Ensaios preliminares}

Os resultados obtidos nos ensaios preliminares foram decisivos para a otimização do processo e encontram-se descritos resumidamente a seguir:

Variação da velocidade de rotação da rosca: com a rosca a uma rotação de 65 rpm e os demais parâmetros fixos, obteve-se uma vazão muito baixa, com pouca descarga de produto. A massa obtida nessa rotação apresentou aparência de um fondant, porém pouco consistente. Observou-se também a formação de alguns cristais grandes (sensíveis ao tato) que, para o caso do fondant, são indesejáveis. O ensaio com a rotação de 100 rpm resultou em uma vazão mais adequada, com cristalização uniforme, observada pela ausência de xarope em meio à massa cristalina e pela textura homogenêa. Observou-se que com 135 rpm o xarope escoava muito rápido pelo cristalizador e a formação de cristais não era aparente. Isto pode indicar que o tempo de residência do xarope no sistema não foi suficiente para induzir eficientemente sua cristalização. Desta forma, a faixa de rotação para o delineamento experimental foi limitada entre de 90-110 rpm.

Temperatura da água de circulação da camisa: nas condições estudadas (10, 15 e $\left.20^{\circ} \mathrm{C}\right)$, observou-se que, com a água de refrigeração entrando a $10^{\circ} \mathrm{C}$ e $15^{\circ} \mathrm{C}$, a rosca travava com frequência, provavelmente em função do endurecimento rápido do xarope em contato com a parede muito fria do equipamento. $\mathrm{Na}$ temperatura de $20^{\circ} \mathrm{C}$, os ensaios foram mais efetivos, não havendo endurecimento do xarope. Como consequência, a temperatura de $20^{\circ} \mathrm{C}$ foi fixada para todos os demais ensaios.

Concentração de xarope de fécula de mandioca na formulação: a sacarose no xarope sem a adição do xarope de fécula de mandioca, dependendo da temperatura utilizada, cristalizava-se rapidamente no reservatório, antes mesmo de entrar na secção da rosca. Com a adição de $5 \%$ de xarope, esse efeito foi diminuído e, com $10 \%$, praticamente não ocorreu. Ao testar concentrações altas de xarope, observou-se que, acima de $20 \%$ de xarope, a rotação do equipamento diminuía, exigindo muito esforço do motor e chegando a travar a rosca em alguns momentos. Dessa forma, ficou estabelecido que a faixa de concentração de xarope utilizada no delineamento experimental seria de 5 a 17\%, gerando experimentos com 0,92 e $21,08 \%$ de xarope, referentes aos pontos axiais do delineamento experimental (Composto Central Rotacional).

Temperatura inicial de cristalização: em temperaturas próximas a $50{ }^{\circ} \mathrm{C}$, o xarope se tornava muito viscoso e apresentava dificuldades para escoar pela válvula de alimentação e atingir a rosca de uma forma contínua, também tendo sido observado um esforço demasiado do motor para manter a velocidade de rotação da rosca constante. O comportamento mecânico do equipamento foi muito bom quando a temperatura na cristalização permanecia entre 60 e $80^{\circ} \mathrm{C}$, sendo esse limite adotado no delineamento experimental.

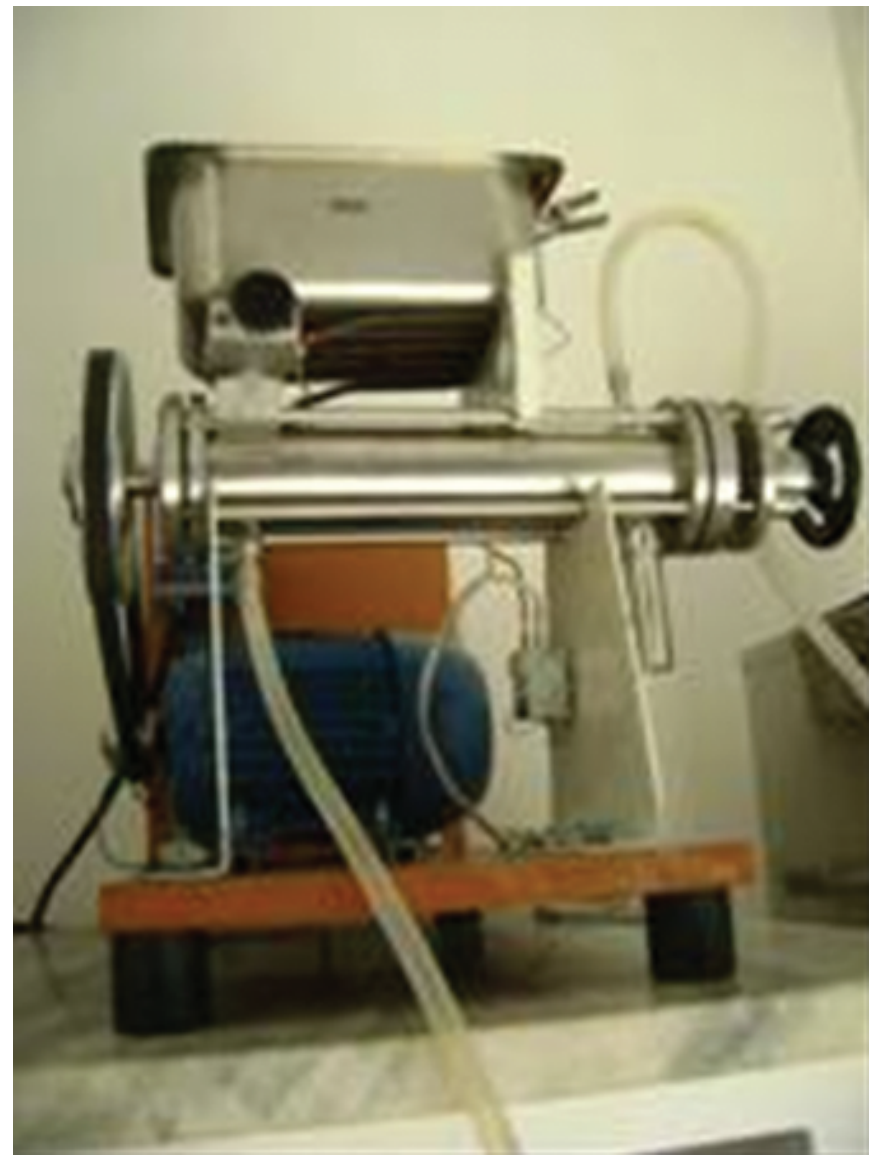

Figura 3. Cristalizador piloto para fondant.
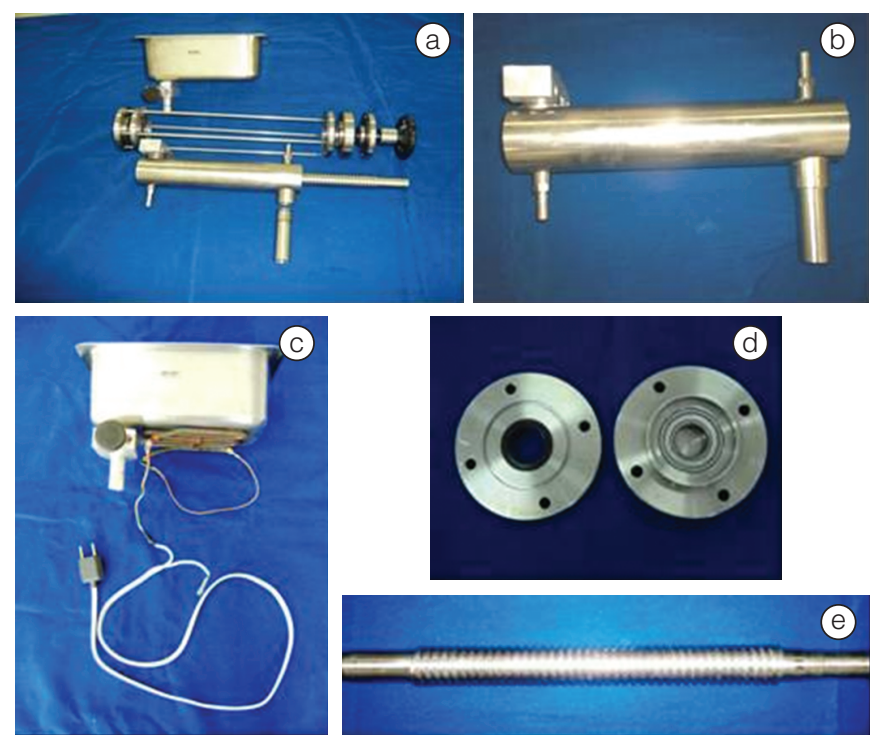

Figura 4. Equipamento desmontado com alguns componentes em evidência: (a) completo; (b) camisa de resfriamento; (c) tanque de alimentação do xarope com resistência elétrica acoplada; (d) encaixe e rotação da rosca; (e) rosca. 


\subsection{Otimização do processo}

A Tabela 3 apresenta os experimentos gerados pelo delineamento experimental, indicando as variáveis reais assim como as repostas de atividade de água e textura (dureza).

Utilizando-se o programa computacional Statistica ${ }^{\circledR}$, determinaram-se os coeficientes da regressão e o coeficiente de correlação $\left(R^{2}\right)$, bem como a análise de variância (ANOVA) para as duas respostas estudadas (Tabela 4).

Para a resposta de atividade de água, para $p<0,05$, somente as variáveis lineares concentração de xarope de glicose $\left(x_{1}\right)$ e velocidade da rosca $\left(x_{2}\right)$ foram estatisticamente significativas. Dessa maneira o modelo que representa a resposta, excluindo-se os efeitos não significativos, é apresentado a seguir:

$y=0,8142-0,0196 x_{1}-0,0017 x_{2}$

Observa-se pelos resultados da ANOVA (Tabela 4) que o modelo proposto se ajusta aos dados experimentais, tendo Fcal para a regressão maior que o Ftab (estatisticamente significativo para $p<0,05$ ). $O$ coeficiente de correlação $R=0,99$ revela ótimo ajuste do modelo matemático aos dados experimentais.

Na Figura 5, é apresentado o gráfico de contorno para o modelo proposto, para os parâmetros significativos para a resposta atividade de água.

Analisando-se o gráfico, é possível observar que a variação da velocidade de rotação da rosca tem pouca influência no valor da atividade de água do fondant. A
Aw é alterada quase que exclusivamente pela variação da porcentagem de xarope do produto. Um aumento da concentração de xarope gerou diminuição na atividade de água dos ensaios.

Dessa forma, sabendo-se que produtos que apresentam valores de atividade de água mais baixos tendem a ter maior estabilidade microbiológica e que valores reportados em literatura para o fondant apontam uma variação de 0,75 a 0,77 (WILLS, 1998), a faixa ótima de trabalho para a concentração de xarope de fécula de mandioca na formulação, neste experimento, deve ser maior ou igual a $20 \%$, o que corresponderá a atividades de água menores ou iguais a 0,79 no estudo realizado.

No caso da resposta de textura (dureza), para $p<0,25$, as variáveis não significativas estatisticamente foram temperatura linear $\left(x_{3}\right)$, velocidade da rosca quadrática $\left(\mathrm{x}_{2}{ }^{2}\right)$, interação entre porcentagem de xarope e velocidade da rosca $\left(x_{1} \cdot x_{2}\right)$, e porcentagem de xarope linear $\left(x_{1}\right)$. Dessa maneira, o modelo proposto, eliminando-se os efeitos não significativos, é apresentado a seguir:

$$
\begin{aligned}
& y=173,44-26,82 x_{1}{ }^{2}+14,02 x_{3}{ }^{2}+ \\
& +25,14 x_{2}-9,62 x_{1} x_{3}+12,15 x_{2} x_{3}
\end{aligned}
$$

Observa-se pelos resultados da ANOVA (Tabela 3) que o modelo de segunda ordem proposto se ajusta aos dados experimentais, tendo Fcal para a regressão maior que o Ftab (estatisticamente significativo para $p<0,25)$. O coeficiente de correlação $R=0,84$ revela

Tabela 3. Delineamento experimental gerado pelo planejamento fatorial completo $2^{3} \mathrm{com}$ pontos axiais e centrais, e repostas para atividade de água e dureza.

\begin{tabular}{cccccc} 
Ensaios & $\begin{array}{c}\text { Variáveis reais } \\
\text { Xe mandioca (\%) }\end{array}$ & $\begin{array}{c}\text { Respostas } \\
\text { Velocidade da } \\
\text { rosca }(\mathbf{r p m})\end{array}$ & $\begin{array}{c}\text { Temperatura de } \\
\left.\text { cristalização } \mathbf{~}^{\circ} \mathbf{C}\right)\end{array}$ & Aw, $\mathbf{2 5}{ }^{\circ} \mathbf{C}$ & Dureza (gf) \\
\hline 1 & 5,00 & 90,0 & 60,0 & $0,836 \pm 0,002$ & $103,1 \pm 5,1$ \\
2 & 5,00 & 90,0 & 80,0 & $0,835 \pm 0,002$ & $106,7 \pm 12,4$ \\
3 & 5,00 & 110,0 & 60,0 & $0,834 \pm 0,002$ & $137,6 \pm 14,2$ \\
4 & 5,00 & 110,0 & 80,0 & $0,831 \pm 0,003$ & $207,5 \pm 23,6$ \\
5 & 17,00 & 90,0 & 60,0 & $0,799 \pm 0,003$ & $133,6 \pm 13,1$ \\
6 & 17,00 & 90,0 & 80,0 & $0,801 \pm 0,002$ & $116,4 \pm 13,5$ \\
7 & 17,00 & 110,0 & 60,0 & $0,793 \pm 0,003$ & $193,9 \pm 23,8$ \\
8 & 17,00 & 110,0 & 80,0 & $0,795 \pm 0,001$ & $207,5 \pm 12,7$ \\
9 & 0,92 & 100,0 & 70,0 & $0,849 \pm 0,004$ & $139,6 \pm 13,4$ \\
10 & 21,08 & 100,0 & 70,0 & $0,778 \pm 0,003$ & $83,7 \pm 14,5$ \\
11 & 11,00 & 83,2 & 70,0 & $0,814 \pm 0,004$ & $175,5 \pm 21,8$ \\
12 & 11,00 & 116,8 & 70,0 & $0,811 \pm 0,002$ & $209,0 \pm 18,8$ \\
13 & 11,00 & 100,0 & 53,2 & $0,812 \pm 0,003$ & $259,0 \pm 19,9$ \\
14 & 11,00 & 100,0 & 86,8 & $0,809 \pm 0,003$ & $194,9 \pm 12,6$ \\
15 & 11,00 & 100,0 & 70,0 & $0,814 \pm 0,003$ & $172,5 \pm 13,7$ \\
16 & 11,00 & 100,0 & 70,0 & $0,815 \pm 0,001$ & $157,8 \pm 17,8$ \\
17 & 11,00 & 100,0 & 70,0 & $0,816 \pm 0,002$ & $175,3 \pm 15,9$ \\
\hline
\end{tabular}


um ajuste satisfatório do modelo matemático aos dados experimentais.

A seguir, são apresentados os gráficos de contorno para o modelo proposto, combinando-se as três variáveis plotadas duas a duas, para a resposta de dureza.

Para uma análise mais significativa do parâmetro de textura, utilizaram-se como referência valores de dureza esperados para um fondant com umidade ao redor de 12 a $13 \%$, encontrados para as amostras do delineamento e tendo-se como base as durezas de fondants de mercado. Para uma umidade de $13 \%$, a dureza encontrada para o fondant foi de 150 gf. Considerou-se, portanto, para esta avaliação, uma faixa de 150 a 200 gf para se obter produtos com características adequadas de textura instrumental na variação de umidade encontrada.

Analisando-se a Figura 6, é possível notar que as variáveis - velocidade de rotação da rosca e porcentagem de xarope de glicose - provocam modificação na textura do fondant. Sob uma mesma velocidade de rotação e variando o teor de xarope, a textura da massa aumenta até cerca de $15 \%$ de xarope e, após esse ponto, passa a diminuir. Mantendo o teor de xarope constante, com o aumento da velocidade de rotação, há um aumento da dureza do fondant.

Com relação à intensidade da agitação, sugere-se que o aumento do movimento leva a uma melhor transferência de massa com formação de uma estrutura semissólida com muitos cristais pequenos, os quais estarão envolvidos pela fase contínua, dando uma resposta de textura mais firme. Agitação mais intensa geralmente resulta em uma nucleação mais rápida e na formação de mais cristais menores, pois ela permite que as moléculas de açúcar migrem mais facilmente e se unam ao cristal latente (HARTEL, 2001).

Valores próximos a 200 gf são obtidos para porcentagem de xarope ao redor do ponto central e velocidades de rotação de 110 rpm ou maiores.
A Figura 7 indica que as variáveis - temperatura de cristalização e porcentagem de xarope - mantêm o mesmo valor de textura na região próxima ao ponto central (aproximadamente 13\% de xarope e cerca de $70{ }^{\circ} \mathrm{C}$ ). Abaixo dessa concentração, a textura diminui; acima, passa a crescer com a elevação da temperatura de cristalização e sob uma mesma concentração de xarope. Já para a segunda variável, o efeito é oposto. A uma mesma temperatura, o valor da textura do fondant aumenta e volta a diminuir com maiores teores de xarope de glicose na formulação do produto. Medidas de dureza na faixa de $200 \mathrm{gf}$ são encontradas em concentrações de 7 a $20 \%$ de xarope e temperaturas abaixo de 60 ou acima de $80^{\circ} \mathrm{C}$

A Figura 8 indica que a dureza aumenta com o aumento da velocidade de rotação, assim como com o

Tabela 4. Resultados da análise estatística para as duas respostas estudadas.

\begin{tabular}{|c|c|c|}
\hline $\begin{array}{l}\text { Coeficientes da regressão } \\
\text { para o modelo de } 2^{\text {a }} \text { ordem }\end{array}$ & $\begin{array}{l}\text { Atividade de } \\
\text { água }\end{array}$ & Dureza \\
\hline$b_{0}$ & 0,8148 & 170,3568 \\
\hline$b_{1}$ & $-0,0392$ & $1,1114^{\star \star}$ \\
\hline$b_{2}$ & $-0,0034$ & 49,5886 \\
\hline$b_{3}$ & $-0,0016^{\star}$ & $-6,2542^{\star \star}$ \\
\hline$b_{11}$ & $0,0005^{\star}$ & $-52,4742$ \\
\hline$b_{22}$ & $-0,0002^{*}$ & $4,6332^{\star \star}$ \\
\hline$b_{33}$ & $-0,00074^{*}$ & 29,2222 \\
\hline$b_{12}$ & $-0,0015^{\star}$ & $5,2000^{\star \star}$ \\
\hline$b_{13}$ & $0,0020^{*}$ & $-18,0800$ \\
\hline$b_{23}$ & $-0,0005^{\star}$ & 23,1200 \\
\hline $\mathrm{R}^{2} / \mathrm{R}$ & $0,98 / 0,99$ & $0,71 / 0,84$ \\
\hline $\begin{array}{l}\text { Valor de F para a regressão } \\
\text { (calculado) }\end{array}$ & 802,71 & 7,18 \\
\hline $\begin{array}{l}\text { Valor de F para a regressão } \\
\text { (tabelado) }\end{array}$ & 4,54 & 1,55 \\
\hline
\end{tabular}

${ }^{*}$ Não significativo para $p<0,05 .{ }^{* *}$ Não significativo para $p<0,2$.

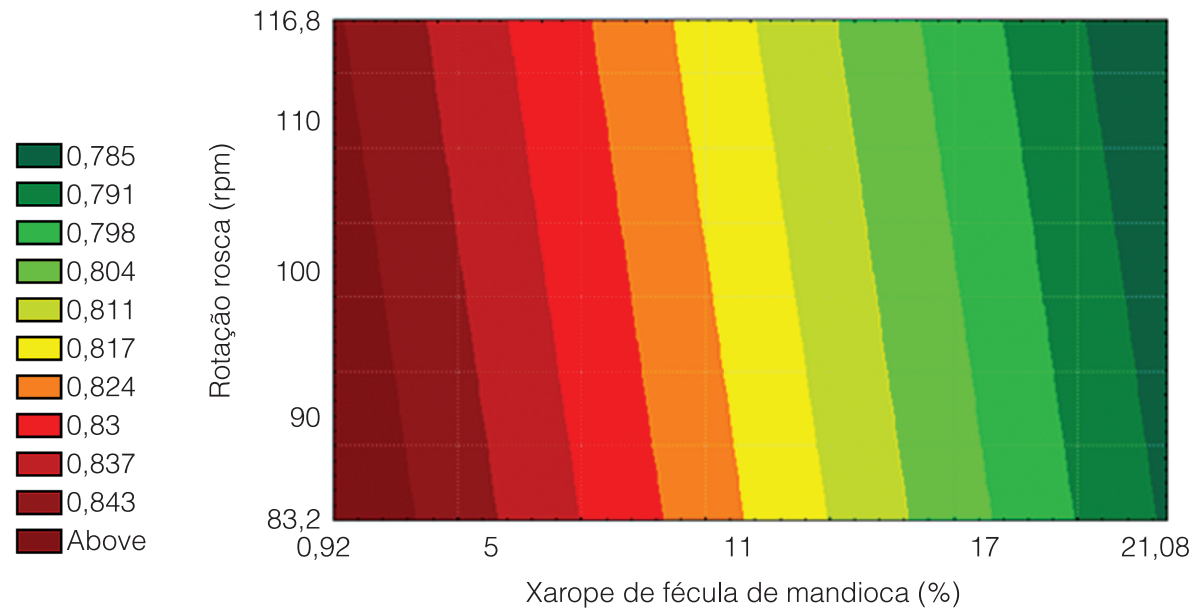

Figura 5. Gráfico de contorno para a Aw dos fondants produzidos em cristalizador contínuo. 


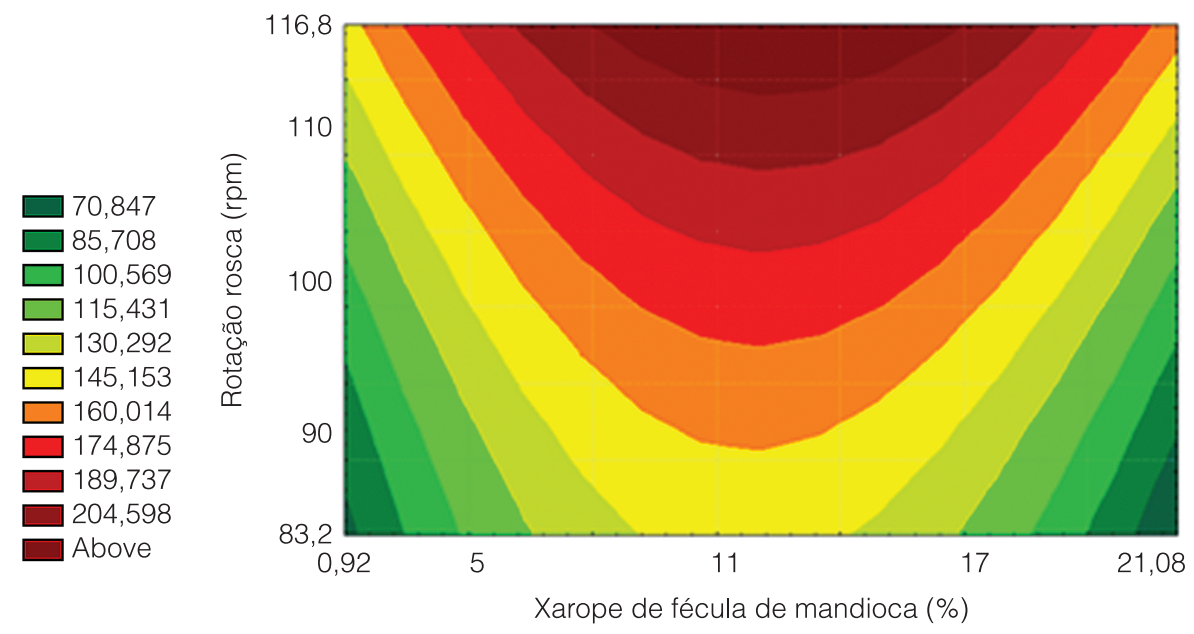

Figura 6. Gráfico de contorno para a dureza dos fondants produzidos em cristalizador contínuo (rotação da rosca × porcentagem de xarope de fécula de mandioca).

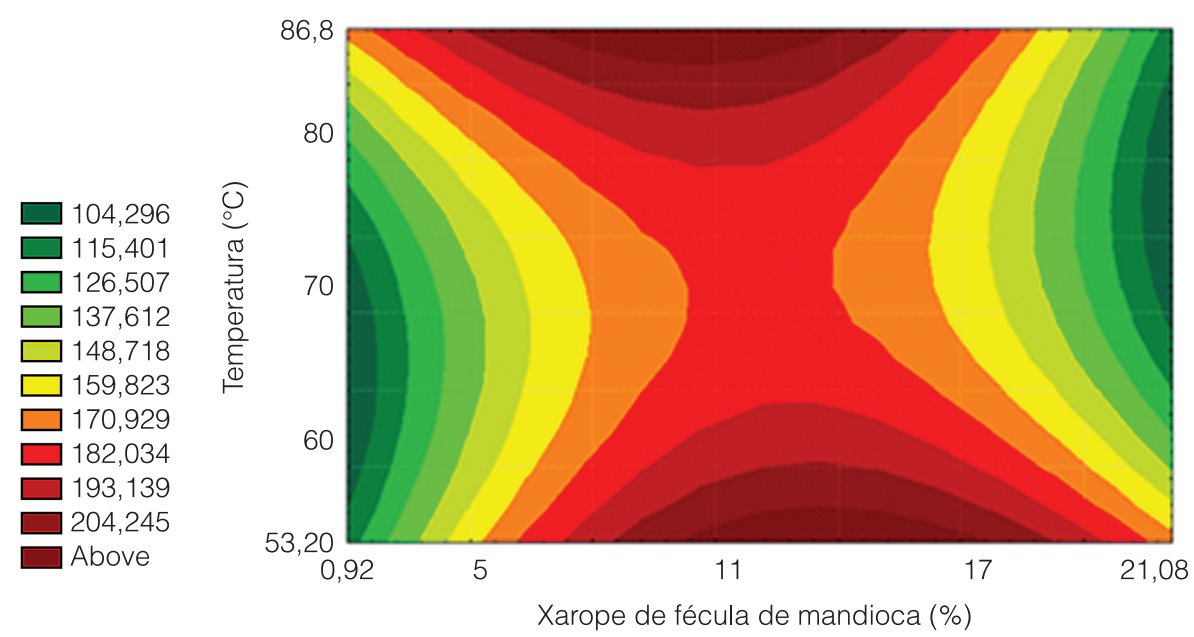

Figura 7. Gráfico de contorno para a dureza dos fondants produzidos em cristalizador contínuo (Porcentagem de xarope $\times$ Temperatura de cristalização).

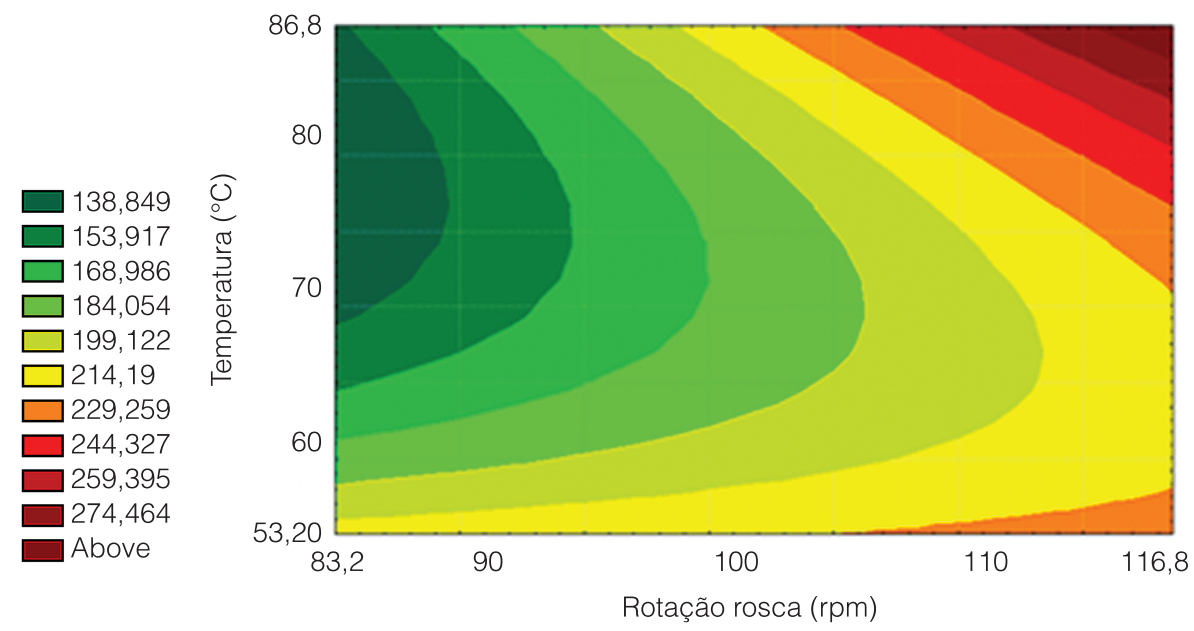

Figura 8. Gráfico de contorno para a dureza dos fondants produzidos em cristalizador contínuo (rotação $\times$ temperatura de cristalização). 
aumento da temperatura. Durezas de 200 gf são obtidas em uma ampla faixa de rotação e de temperatura, mas se tornam maiores nas regiões de maiores rotações e maiores temperaturas $\left(110 \mathrm{rpm} \mathrm{e} 80^{\circ} \mathrm{C}\right)$.

\section{Conclusão}

O cristalizador construído apresentou um bom desempenho do ponto de vista de obtenção de massas cristalinas com características similares a produtos do tipo fondant de açúcar encontrados no mercado. A partir de sua construção e colocação em operação, foi possível estabelecer e estudar algumas condições de processo para a cristalização em condições dinâmicas, simulando uma produção industrial.

Os resultados para a otimização do processo evidenciaram que a atividade de água tem maior influência conforme porcentagem de xarope na formulação, diminuindo com o aumento desta, tendo valores próximos aos reportados na literatura para o tipo de produto com $20 \%$ de adição deste ingrediente. Este resultado está de acordo com o previsto, pois concentrações maiores de xarope geram produtos com maiores concentrações de sólidos na fase líquida, já que esta fase conterá toda a água, todo o xarope e suficiente quantidade de sacarose para manter o equilíbrio de saturação da fase. Não se esperava que a velocidade de rotação nem a temperatura inicial de cristalização tivessem alguma influência sobre a atividade de água do fondant, uma vez que este parâmetro tem relação direta com a concentração da fase líquida, que não deve ser afetada pelas condições de processo (agitação e temperatura) e sim pela composição do xarope.

A textura sofre influência de todas as variáveis estudadas e, mais intensamente, da velocidade de rotação e do teor de xarope de mandioca; apresenta valores otimizados para porcentagem de xarope de fécula de mandioca acima de $13 \%$, velocidade de rotação em torno de $110 \mathrm{rpm}$ e temperatura inicial de cristalização de 60 a $80^{\circ} \mathrm{C}$.

Considerando-se ambas as respostas na otimização, conclui-se que, para obter as melhores características no fondant, ou seja, textura adequada e maior estabilidade microbiológica, deve-se trabalhar com teores de xarope de glicose de, no mínimo, 20\% (mínima atividade de água), velocidade de rotação entre 105 e 110 rpm, e temperatura inicial de cristalização ao redor de $60^{\circ} \mathrm{C}$.

\section{Referências}

BARROS NETO, B.; SCARMINIO, I. S.; BRUNS, R.E. Como Fazer Experimentos: Pesquisa e Desenvolvimento na Ciência e na Indústria. Campinas: Editora da Unicamp, 2001. 401 p.

BOX, G. E. P.; HUNTER, J. S.; STUART, J. S. Statistics For Experimenters. New York: John Wiley \& Sons, 1978. 657 p.

HARTEL, R. W. Crystallization in Foods. Gaithersburg: Aspen Publishers Inc., 2001. 325 p. (Aspen Food Engineering Series).

HARTEL, R. W.; SHASTRY, A. Sugar crystallization in foods products. Critical Reviews in Food Science and Nutrition, London, v. 30, n. 1, p.49-112, 1991. http://dx.doi. org/10.1080/10408399109527541

JEFFERY, M. S. Centers, fondants, marzipans and crystallized confectionery. In: JACKSON, E. B. (Ed.). Sugar Confectionery Manufacture. London: Blackie Academic \& Professional, 1993. p. $280-299$

JEFFERY, M. S. Grained and ungrained confections. The Manufacturing Confectioner, Glen Rock, v. 81, n. 6, p. 110, 2001.

QUEIROZ, M. B.; BRAÚNA, I. R. L.; KIECKBUSCH, T. G. Sucrose Crystallization from a Multicomponent Solution using Fondant as Model System.In: INTERNATIONAL CONFERENCE FOR CONVEYING AND HANDLING OF PARTICULATE SOLIDS, 5., 2006, Sorrento, Italy. Anais... Sorrento: Ortra Ltd., 2006.

VILCHEZ, J.; JOCH,B.; ROCZNIK,D. Device and method for producing fondant. Patent Application Patent Application Publication. US 2006, 0045952A1, 2006.

WILLS, D. Water activity and its importance in making candy. The Manufacturing Confectioner, Glen Rock, v. 78, n. 8 , p. 71-74, 1998. 